\title{
Convergence of Relaxed Matrix Parallel Multisplitting Chaotic Methods for $H$-Matrices
}

\author{
Li-Tao Zhang, ${ }^{1,2}$ Jian-Lei Li, ${ }^{3}$ Tong-Xiang Gu, ${ }^{2}$ and Xing-Ping Liu ${ }^{2}$ \\ ${ }^{1}$ Department of Mathematics and Physics, Zhengzhou Institute of Aeronautical Industry Management, Zhengzhou, \\ Henan 450015, China \\ ${ }^{2}$ Laboratory of Computationary Physics, Institute of Applied Physics and Computational Mathematics, \\ P.O. Box 8009, Beijing 100088, China \\ ${ }^{3}$ College of Mathematics and Information Science, North China University of Water Resources and Electric Power, \\ Zhengzhou, Henan 450011, China
}

Correspondence should be addressed to Li-Tao Zhang; litaozhang@163.com

Received 9 May 2014; Accepted 30 June 2014; Published 17 July 2014

Academic Editor: Shuqian Shen

Copyright (C) 2014 Li-Tao Zhang et al. This is an open access article distributed under the Creative Commons Attribution License, which permits unrestricted use, distribution, and reproduction in any medium, provided the original work is properly cited.

Based on the methods presented by Song and Yuan (1994), we construct relaxed matrix parallel multisplitting chaotic generalized USAOR-style methods by introducing more relaxed parameters and analyze the convergence of our methods when coefficient matrices are $\mathrm{H}$-matrices or irreducible diagonally dominant matrices. The parameters can be adjusted suitably so that the convergence property of methods can be substantially improved. Furthermore, we further study some applied convergence results of methods to be convenient for carrying out numerical experiments. Finally, we give some numerical examples, which show that our convergence results are applied and easily carried out.

\section{Introduction}

For solving the large sparse linear system

$$
A x=b \text {, }
$$

where $A$ is an $n \times n$ real nonsingular matrix and $x, b \in R^{n}$, an iterative method is usually considered. The concept of matrix multisplitting solution of linear system was introduced by O'Leary and White [1] and further studied by many other authors. Frommer and Mayer [2] studied extrapolated relaxed matrix multisplitting methods and matrix multisplitting SOR method. Mas et al. [3] analyzed nonstationary extrapolated relaxed and asynchronous relaxed methods. Gu et al. [4, 5] further studied relaxed nonstationary two-stage matrix multisplitting methods and the corresponding asynchronous schemes.

As we know, matrix multisplitting iterative method for linear systems takes two basic forms. When all of the processors wait until they are updated with the results of the current iteration, it is synchronous. That is to say, when they begin the next iteration of asynchronous, they may act more or less independently of each other and use possibly delayed iterative values of the output of the other processors. In view of the potential time saving inherent in them, asynchronous iterative methods, or chaotic as they are often called, have attracted much attention since the early paper of Chazan and Miranker [6] introduced them in the context of point iterative schemes. Naturally, a number of convergence results [7-21] have been obtained. Recently, the convergence of three relaxed matrix multisplitting chaotic AOR methods has been investigated in $[11,13,14]$.

A collection of triples $\left(M_{l}, N_{l}, E_{l}\right), l=1,2, \ldots, s$, is called a multisplitting of $A$ if $A=M_{l}-N_{l}$ is a splitting of $A$ for $l=$ $1,2, \ldots, s$, and $E_{l}$ 's, called weighting matrices, are nonnegative diagonal matrices such that $\sum_{l=1}^{s} E_{l}=I$.

The unsymmetric accelerated overrelaxation (USAOR) method was proposed in [22], if the diagonal elements of the matrix $A$ are nonzero. Without the loss of generality, let the matrix $A$ be split as

$$
A=I-L-U,
$$


where $I, L$, and $U$ are nonsingular diagonal, strictly lower triangular and upper triangular parts of $A$. The iterative scheme of the USAOR method is defined by

$$
\begin{aligned}
x^{(k+(1 / 2))} & =H\left(\omega_{1}, \omega_{2}\right) x^{(k)}+\omega_{1}\left(I-\omega_{2} L\right)^{-1} b, \\
x^{(k+1)} & =H\left(\omega_{3}, \omega_{4}\right) x^{(k+(1 / 2))}+\omega_{3}\left(I-\omega_{4} U\right) b ;
\end{aligned}
$$

that is,

$$
x^{(k+1)}=H_{\mathrm{USAOR}} x^{(k)}+G_{\mathrm{USAOR}}, \quad k=0,1, \ldots,
$$

where

$$
\begin{gathered}
H_{\text {USAOR }}=H\left(\omega_{3}, \omega_{4}\right) H\left(\omega_{1}, \omega_{2}\right), \\
H\left(\omega_{1}, \omega_{2}\right)=\left(I-\omega_{2} L\right)^{-1} \\
\times\left[\left(1-\omega_{1}\right) I+\left(\omega_{1}-\omega_{2}\right) L+\omega_{1} U\right], \\
H\left(\omega_{3}, \omega_{4}\right)=\left(I-\omega_{4} U\right)^{-1}\left[\left(1-\omega_{3}\right) I+\left(\omega_{3}-\omega_{4}\right) U+\omega_{3} L\right], \\
G_{\text {USAOR }}=\left(I-\omega_{4} U\right)^{-1}\left[\left(\omega_{1}+\omega_{3}-\omega_{1} \omega_{3}\right) I+\omega_{3}\left(\omega_{1}-\omega_{2}\right) L\right. \\
\left.+\omega_{1}\left(\omega_{3}-\omega_{4}\right) U\right]\left(I-\omega_{2} L\right)^{-1} b,
\end{gathered}
$$

and $\alpha_{i}, \beta_{i}(i=1,2)$ are real parameters.

In this paper, we will extend the point USAOR iterative method to matrix multisplitting chaotic generalized USAOR method and analyze their convergence for $H$-matrices and irreducible diagonally dominant matrices.

Based on matrix multisplitting chaotic generalized AOR method [23], the matrix multisplitting chaotic generalized USAOR method is given by

$$
x^{(k+1)}=H_{\mathrm{GUSAOR}} x^{(k)}+G_{\mathrm{GUSAOR}}, \quad k=0,1,2, \ldots,
$$

where

$$
\begin{gathered}
H_{\mathrm{GUSAOR}}=H(\beta, \psi) H(\alpha, \phi) \\
H(\alpha, \phi)=(I-\alpha \phi L)^{-1}[I-\phi+(1-\alpha) \phi L+\phi U] \\
H(\beta, \psi)=(I-\beta \psi U)^{-1}[I-\psi+(1-\beta) \psi U+\psi L], \\
G_{\mathrm{GUSAOR}}=(1-\beta \psi U)^{-1}[2 I-\psi+(\psi-\alpha \phi) L \\
+(1-\beta) \psi U](I-\alpha \phi L)^{-1} b
\end{gathered}
$$

is an iteration matrix and $\phi=\operatorname{diag}\left(\beta_{1}, \beta_{2}, \ldots, \beta_{n}\right), \psi=$ $\operatorname{diag}\left(\gamma_{1}, \gamma_{2}, \ldots, \gamma_{n}\right)$ with $\beta_{i} \geq 0, \gamma_{i} \geq 0(i=1,2, \ldots, n)$ and $\alpha, \beta$ real parameters.

Remark 1. For $\beta=\gamma_{i}=0, i=1,2, \ldots, n$, then GUSAOR method reduces to GAOR method [11]; For $\phi=\omega_{1} I, \alpha=$ $\omega_{2} / \omega_{1}, \psi=\omega_{3} I, \beta=\omega_{4} / \omega_{3}$, the GUSAOR method reduces to the USAOR method [22].

The remainder of this paper is organized as follows. In Section 2, we introduce some notations and preliminaries. In Section 3, we present relaxed matrix parallel multisplitting chaotic GUSAOR-style methods for solving large nonsingular system, when the coefficient matrix $A$ is an $H$-matrix or irreducible diagonally dominant matrix, and analyze the convergence of our methods. In Section 4, we further study some applied convergence results of methods to be convenient for carrying out numerical experiments. In Section 5, we give some numerical examples, which show that our convergence results are easily carried out. Finally, we draw some conclusions.

\section{Notation and Preliminaries}

We will use the following notation. Let $C=\left(c_{i j}\right) \in R^{n \times n}$ be an $n \times n$ matrix. By $\operatorname{diag}(C)$ we denote the diagonal matrix coinciding in its diagonal with $C$. For $A=\left(a_{i j}\right), B=\left(b_{i j}\right) \in$ $R^{n \times n}$, we write $A \geq B$ if $a_{i j} \geq b_{i j}$ holds for all $i, j=1,2, \ldots, n$. Calling $A$ nonnegative if $A \geq 0$, we say that $B \leq C$ if and only if $-B \geq-C$. These definitions carry immediately over to vectors by identifying them with $n \times 1$ matrices. By $|A|=\left(\left|a_{i j}\right|\right)$ we define the absolute value of $A \in R^{n \times n}$. We denote by $\langle A\rangle=$ $\left(\left\langle a_{i j}\right\rangle\right)$ the comparison matrix of $A \in R^{n \times n}$ where $\left\langle a_{i j}\right\rangle=\left|a_{i j}\right|$ for $i=j$ and $\left\langle a_{i j}\right\rangle=-\left|a_{i j}\right|$ for $i \neq j, i, j=1,2, \ldots, n$. Spectral radius of a matrix $A$ is denoted by $\rho(A)$. It is well known that if $A \geq 0$ and there exists a vector $x>0$ such that $A x<\alpha x$, then $\rho(A)<\alpha$.

Definition 2 (see [24]). Let $A \in R^{n \times n}$. It is called an

(1) $L$-matrix if $a_{i i}>0$ for $i=1,2, \ldots, n$, and $a_{i j} \leq 0$ for $i \neq j, i, j=1,2, \ldots, n$;

(2) $M$-matrix if it is a nonsingular $L$-matrix satisfying $A^{-1} \geq 0$

(3) $H$-matrix if $\langle A\rangle$ is an $M$-matrix.

Lemma 3 (see [13]). If $A$ is an $H$-matrix, then

(1) $\left|A^{-1}\right| \leq\langle A\rangle^{-1}$;

(2) there exists a diagonal matrix $P$ whose diagonal entries are positive such that $\langle A\rangle P e>0$ withe $=(1,1, \ldots, 1)^{T}$.

Lemma 4 (see [13]). Let $A$ be an $M$-matrix and let the splitting $A=M-N$ be an $M$-splitting. If $P$ is the diagonal matrix defined in Lemma 3, then $\left\|P^{-1} M^{-1} N P\right\|_{\infty}<1$.

Finally, a sequence of sets $P_{i}$ with $P_{i} \subseteq\{1, \ldots, s\}$ is admissible if every integer $1,2, \ldots, s$ appears infinitely often in the $P_{i}$, while such an admissible sequence is regulated if there exists a positive integer $T$ such that each of the integers appears at least once in any $T$ consecutive sets of the sequence.

\section{Relaxed Matrix Multisplitting Parallel Chaotic Methods}

Using the given models in $[7,11,13]$ and (4), we may describe the corresponding three algorithms of relaxed matrix multisplitting chaotic GUSAOR-style methods, which are as follows. 
Algorithm 5 (given the initial vector). For $k=0,1, \ldots$, until convergence. Parallel computing the following iterative scheme

$$
\begin{gathered}
x^{(k+1)}=\sum_{l=1}^{s} E_{l}\left(H_{\mathrm{GUSAOR}}\right)_{l}^{\mu_{l, k}}\left(x^{(k)}\right) \\
\left(H_{\mathrm{GUSAOR}}\right)_{l}\left(x^{(k)}\right)=H(\beta, \psi)_{l} H(\alpha, \phi)_{l} x^{(k)}+\left(G_{\mathrm{GUSAOR}}\right)_{l} b \\
H(\alpha, \phi)_{l}=\left(I-\alpha \phi L_{l}\right)^{-1}\left[I-\phi+(1-\alpha) \phi L_{l}+\phi U_{l}\right] \\
H(\beta, \psi)_{l}=\left(I-\beta \psi U_{l}\right)^{-1}\left[I-\psi+(1-\beta) \psi U_{l}+\psi L_{l}\right] \\
\left(G_{\mathrm{GUSAOR}}\right)_{l}=\left(I-\beta \psi U_{l}\right)^{-1} \\
\times\left[2 I-\psi+(\psi-\alpha \phi) L_{l}+(1-\beta) \psi U_{l}\right] \\
\times\left(I-\alpha \phi L_{l}\right)^{-1}
\end{gathered}
$$

with $\alpha \geq 0, \beta \geq 0, \beta_{i}>0, \gamma_{i}>0$, and $\mu_{l, k} \geq 1$, where $\left(H_{\mathrm{GUSAOR}}\right)_{l}^{\mu_{l, k}}$ is the $\mu_{l, k}$ th composition of the affine mapping satisfying

$$
\begin{gathered}
\left(H_{\mathrm{GUSAOR}}\right)_{l}^{\mu_{l, k}}= \begin{cases}\left(H_{\mathrm{GUSAOR}}\right)_{l} \cdots\left(H_{\mathrm{GUSAOR}}\right)_{l}, & \mu_{l, k} \geq 1, \\
I, & \mu_{l, k}=0,\end{cases} \\
B=L_{l}+U_{l}, \quad l=1,2, \ldots, s .
\end{gathered}
$$

Remark 6. In Algorithm 5, by using a suitable positive relaxed parameter $\omega$, then we can get the following relaxed Algorithm 7.

Algorithm 7 (given the initial vector). For $k=0,1, \ldots$, until convergence. Parallel computing the following iterative scheme

$$
x^{(k+1)}=\omega \sum_{l=1}^{s} E_{l}\left(H_{\mathrm{GUSAOR}}\right)_{l}^{\mu_{l, k}}\left(x^{(k)}\right)+(1-\omega) x^{(k)},
$$

with $\omega>0, \alpha \geq 0, \beta \geq 0, \beta_{i}>0, \gamma_{i}>0$, and $\mu_{l, k} \geq 1$, where $\left(H_{\mathrm{GUSAOR}}\right)_{l}^{\mu_{l, k}}\left(x^{(k)}\right)$ is defined such as Algorithm 5.

Remark 8. In Algorithm 7, we assume that the index sequence $\left\{P_{i}\right\}$ is admissible and regulated; then we can get the following Algorithm 9 with the case of relaxed chaotic GUSAOR method.

Algorithm 9 (given the initial vector). For $k=0,1, \ldots$, until convergence. Parallel computing the following iterative scheme

$$
\begin{aligned}
x^{(k+1)}= & (I-\omega) \sum_{l \in P_{i}} E_{l} \\
& +\omega \sum_{l \in P_{i}} E_{l}\left(H_{\mathrm{GUSAOR}}\right)_{l}^{\mu_{l, k}}\left(x^{\left(k-r_{k}+1\right)}\right),
\end{aligned}
$$

with $\omega>0, \alpha \geq 0, \beta \geq 0, \beta_{i}>0, \gamma_{i}>0, \mu_{l, k} \geq 1$, and $P_{i} \subseteq\{1, \ldots, s\}$, where $\left(H_{\mathrm{GUSAOR}}\right)_{l}\left(x^{(k)}\right)$ of Algorithm 5 are replaced by $\left(H_{\mathrm{GUSAOR}}\right)_{l}\left(x^{\left(k-r_{k}+1\right)}\right)$ and $x^{\left(k-r_{k}+1\right)}=$ $\left(x_{1}^{(k-r(1, k))}, x_{2}^{(k-r(2, k))}, \ldots, x_{n}^{(k-r(n, k))}\right)^{T}$.
Remark 10. Relaxed matrix multisplitting chaotic GUSAORstyle methods introduce more relaxed factors, so our methods are the generalization of $[5,12,13]$. The parameters can be adjusted suitably so that the convergence property of method can be substantially improved.

Using Lemmas 3 and 4, we can get the following convergence result according to Algorithm 5.

Theorem 11. Let $A \in R^{n \times n}$ be an $H$-matrix and $\left(I-L_{l}, U_{l}, E_{l}\right)$, $l=1,2, \ldots, s$, a multisplitting of $A$. Assume that for $l=$ $1,2, \ldots, s$, we have the following.

(1) $L_{l}$ are the strictly lower triangular matrices and $U_{l}$ are the matrices such that the equalities $A=I-L_{l}-U_{l}$ hold.

(2) $\langle A\rangle=|I|-\left|L_{l}\right|-\left|U_{l}\right|=|I|-|B|$, where $|B|=\left|L_{l}\right|+\left|U_{l}\right|$.

Then the sequence $\left\{x^{(k)}\right\}$ generated by Algorithm 5 converges for any initial $x^{(0)}$ if and only if $\left(\alpha, \beta, \beta_{i}, \gamma_{i}\right), i=1,2, \ldots n \in W_{1}$, $\rho=(|B|)$, where

$$
\begin{aligned}
W_{1}=\left\{\left(\alpha, \beta, \beta_{i}, \gamma_{i}\right) \in R^{4}: 0 \leq \alpha, \beta \leq 1,\right. \\
\left.\quad 0<\beta_{i}, \gamma_{i}<\frac{2}{1+\rho}, i=1,2, \ldots, n\right\} .
\end{aligned}
$$

Proof. Define the iteration matrix in Algorithm 5

$$
\left(H_{\mathrm{GUSAOR}}\right)_{k}=\sum_{l=1}^{s} E_{l}\left\{H(\beta, \psi)_{l} H(\alpha, \phi)_{l}\right\}^{\mu_{l, k}},
$$

where

$$
\begin{aligned}
& H(\alpha, \phi)_{l}=\left(I-\alpha \phi L_{l}\right)^{-1}\left[I-\phi+(1-\alpha) \phi L_{l}+\phi U_{l}\right] \\
& H(\beta, \psi)_{l}=\left(I-\beta \psi U_{l}\right)^{-1}\left[I-\psi+(1-\beta) \psi U_{l}+\psi L_{l}\right] .
\end{aligned}
$$

Obviously, we have to find a constant $\sigma$ with $0 \leq \sigma<1$ and some norm, which are independent of $k$, such that for $k \geq 1$, $H_{\mathrm{GUSAOR}}(\alpha, \beta, \phi, \psi)_{k} \| \leq \sigma$.

We first note that the matrices $I-\alpha \phi L_{l}$ and $I-\beta \psi U_{l}$ are $H$-matrices for $l=1,2, \ldots, s$. Thus by Lemma 3 we have the following inequalities:

$$
\begin{aligned}
& \left|\left(I-\alpha \phi L_{l}\right)^{-1}\right| \leq\left\langle I-\alpha \phi L_{l}\right\rangle^{-1}=\left(I-\alpha \phi\left|L_{l}\right|\right)^{-1}, \\
& \left|\left(I-\beta \psi U_{l}\right)^{-1}\right| \leq\left\langle I-\beta \psi U_{l}\right\rangle^{-1}=\left(I-\beta \psi\left|U_{l}\right|\right)^{-1} .
\end{aligned}
$$

From this relation, it follows that

$$
\begin{aligned}
\left|H(\beta, \psi)_{l} H(\alpha, \phi)_{l}\right|= & \mid\left(I-\beta \psi U_{l}\right)^{-1} \\
& \times\left[I-\psi+(1-\beta) \psi U_{l}+\psi L_{l}\right] \\
& \times\left(I-\alpha \phi L_{l}\right)^{-1} \\
& \times\left[I-\phi+(1-\alpha) \phi L_{l}+\phi U_{l}\right] \mid
\end{aligned}
$$




$$
\begin{aligned}
\leq & \left\langle I-\beta \psi U_{l}\right\rangle^{-1} \\
& \times\left|I-\psi+(1-\beta) \psi U_{l}+\psi L_{l}\right| \\
& \times\left\langle I-\alpha \phi L_{l}\right\rangle^{-1} \\
& \times\left|I-\phi+(1-\alpha) \phi L_{l}+\phi U_{l}\right| \\
\leq & \left(I-\beta \psi\left|U_{l}\right|\right)^{-1} \\
& \times\left[|I-\psi|+|1-\beta| \psi\left|U_{l}\right|+\psi\left|L_{l}\right|\right] \\
& \times\left(I-\alpha \phi\left|L_{l}\right|\right)^{-1} \\
& \times\left[|I-\phi|+|1-\alpha| \phi\left|L_{l}\right|+\phi\left|U_{l}\right|\right] .
\end{aligned}
$$

Case 1. Let $0<\beta_{i} \leq 1,0<\gamma_{i} \leq 1, i=1,2, \ldots, n$. Then

$$
\begin{aligned}
& |I-\psi|+|1-\beta| \psi\left|U_{l}\right|+\psi\left|L_{l}\right| \\
& \quad=I-\psi+\psi\left|U_{l}\right|-\beta \psi\left|U_{l}\right|+\psi\left|L_{l}\right| . \\
& |I-\phi|+|1-\alpha| \phi\left|L_{l}\right|+\phi\left|U_{l}\right| \\
& \quad=I-\phi+\phi\left|L_{l}\right|-\alpha \phi\left|L_{l}\right|+\phi\left|U_{l}\right| .
\end{aligned}
$$

Define

$$
\begin{gathered}
M_{l}^{1}(\beta, \psi)=I-\beta \psi\left|U_{l}\right|, \quad M_{l}^{2}(\alpha, \phi)=I-\alpha \phi\left|L_{l}\right|, \\
N_{l}^{1}(\beta, \psi)=|I-\psi|+(1-\beta) \psi\left|U_{l}\right|+\psi\left|L_{l}\right| \\
N_{l}^{2}(\alpha, \phi)=|I-\phi|+(1-\alpha) \phi\left|L_{l}\right|+\phi\left|U_{l}\right| .
\end{gathered}
$$

So, for $l=1,2, \ldots, s$, we have the following relations

$$
\begin{aligned}
M_{l}^{1}(\beta, \psi)-N_{l}^{1}(\beta, \psi) & =\psi-\psi\left|U_{l}\right|-\psi\left|L_{l}\right| \\
& =\psi(I-|B|) . \\
M_{l}^{2}(\alpha, \phi)-N_{l}^{2}(\alpha, \phi) & =\phi-\phi\left|L_{l}\right|-\phi\left|U_{l}\right| \\
& =\phi(I-|B|) .
\end{aligned}
$$

Since for $l=1,2, \ldots, s, M_{l}^{1}(\beta, \psi)$ and $M_{l}^{2}(\alpha, \phi)$ are both $M$-matrices and $N_{l}^{1}(\beta, \psi) \geq 0, N_{l}^{2}(\alpha, \phi) \geq 0$, the splittings $M_{l}^{1}(\beta, \psi)-N_{l}^{1}(\beta, \psi)$ and $M_{l}^{2}(\alpha, \phi)-N_{l}^{2}(\alpha, \phi)$ are $M$-splittings of $\psi(I-|B|)$ and $\phi(I-|B|)$, respectively, which are $M$-matrices. So, from Lemma 4 we may complete the proving courses, which are listed subsequently.

Case 2. Let $1<\beta_{i}<2 /(1+\rho), 1<\gamma_{i}<2 /(1+\rho), i=1,2, \ldots, n$.

Assume that $\beta_{\max }=\max _{1 \leq i \leq n}\left\{\beta_{i}\right\}, \gamma_{\max }=\max _{1 \leq i \leq n}\left\{\gamma_{i}\right\}$. Then we have

$$
\begin{aligned}
& |I-\psi|+(1-\beta) \psi\left|U_{l}\right|+\psi\left|L_{l}\right| \\
& \quad \leq\left(\gamma_{\max }-1\right) I+(1-\beta) \gamma_{\max }\left|U_{l}\right|+\gamma_{\max }\left|L_{l}\right|
\end{aligned}
$$

$$
\begin{aligned}
&|I-\phi|+(1-\alpha) \phi\left|L_{l}\right|+\phi\left|U_{l}\right| \\
& \leq\left(\beta_{\max }-1\right) I+(1-\alpha) \beta_{\max }\left|L_{l}\right|+\beta_{\max }\left|U_{l}\right| \\
&\left(I-\beta \psi\left|U_{l}\right|\right)^{-1} \leq\left(I-\beta \gamma_{\max }\left|U_{l}\right|\right)^{-1} \\
&\left(I-\alpha \phi\left|L_{l}\right|\right)^{-1} \leq\left(I-\alpha \beta_{\max }\left|L_{l}\right|\right)^{-1}
\end{aligned}
$$

Similar to Case 1, we define

$$
\begin{aligned}
& M_{l}^{3}\left(\beta, \gamma_{\max }\right)=I-\beta \gamma_{\max }\left|U_{l}\right|, \\
& M_{l}^{4}\left(\alpha, \beta_{\max }\right)=I-\alpha \beta_{\max }\left|L_{l}\right|, \\
& N_{l}^{3}\left(\beta, \gamma_{\max }\right)=\left(\gamma_{\max }-1\right) I+(1-\beta) \gamma_{\max }\left|U_{l}\right|+\gamma_{\max }\left|L_{l}\right|, \\
& N_{l}^{4}\left(\alpha, \beta_{\max }\right)=\left(\beta_{\max }-1\right) I+(1-\alpha) \beta_{\max }\left|L_{l}\right|+\beta_{\max }\left|U_{l}\right| .
\end{aligned}
$$

Then

$$
\begin{aligned}
& M_{l}^{3}\left(\beta, \gamma_{\max }\right)-N_{l}^{3}\left(\beta, \gamma_{\max }\right)=(2-\beta) I-\beta|B|, \\
& M_{l}^{4}\left(\alpha, \beta_{\max }\right)-N_{l}^{4}\left(\alpha, \beta_{\max }\right)=(2-\alpha) I-\alpha|B| .
\end{aligned}
$$

It is clear, $(2-\beta) I-\beta|B|$ and $(2-\alpha) I-\alpha|B|$ are both $M$-matrices. Since, for $l=1,2, \ldots, s, M_{l}^{1}(\beta, \psi)$ and $M_{l}^{2}(\alpha, \phi)$ are $M$-matrices, $N_{l}^{1}(\beta, \psi) \geq 0$ and $N_{l}^{2}(\alpha, \phi) \geq 0$, the splittings $M_{l}^{1}(\beta, \psi)-N_{l}^{1}(\beta, \psi)$ and $M_{l}^{2}(\alpha, \phi)-N_{l}^{2}(\alpha, \phi)$ are $M$-splittings of the matrices $(2-\beta) I-\beta|B|$ and $(2-\alpha) I-\alpha|B|$, respectively.

Thus, for Cases 1 and 2, from Lemma 4, we have

$$
\begin{gathered}
\left\|P_{1}^{-1}\left(M_{l}^{1}\right)^{-1}(\beta, \psi) N_{l}^{1}(\beta, \psi) P_{1}\right\|_{\infty}<1, \quad l=1,2, \ldots, s, \\
\left\|P_{2}^{-1}\left(M_{l}^{2}\right)^{-1}(\alpha, \phi) N_{l}^{2}(\alpha, \phi) P_{2}\right\|_{\infty}<1, \quad l=1,2, \ldots, s,
\end{gathered}
$$

So

$$
\begin{aligned}
& P_{1}^{-1}\left|\left(H_{\mathrm{GUSAOR}}\right)_{k}\right| P_{1} e \\
& =\sum_{l=1}^{s} E_{l}\left\{P_{1}^{-1} H(\beta, \psi)_{l} H(\alpha, \phi)_{l} P_{1}\right\}^{\mu_{l, k}} e \\
& \leq \sum_{l=1}^{s} E_{l}\left\{P_{1}^{-1}\left(M_{l}^{1}\right)^{-1}(\beta, \psi) N_{l}^{1}(\beta, \psi)\left(M_{l}^{2}\right)^{-1}\right. \\
& \left.\quad \times(\alpha, \phi) N_{l}^{2}(\alpha, \phi) P_{1}\right\}^{\mu_{l, k}} e \\
& =\sum_{l=1}^{s} E_{l}\left\{P_{1}^{-1}\left(M_{l}^{1}\right)^{-1}(\beta, \psi) N_{l}^{1}(\beta, \psi)\right. \\
& \quad \times P_{1} P_{1}^{-1} P_{2} P_{2}^{-1}\left(M_{l}^{2}\right)^{-1} \\
& \left.\quad \times(\alpha, \phi) N_{l}^{2}(\alpha, \phi) P_{2} P_{2}^{-1} P_{1}\right\}^{\mu_{l, k}} e \\
& =\max _{1 \leq l \leq s}\left\{\left\|P_{1}^{-1}\left(M_{l}^{1}\right)^{-1}(\beta, \psi) N_{l}^{1}(\beta, \psi) P_{1}\right\|_{\infty}\right. \\
& \left.\times\left\|P_{2}^{-1}\left(M_{l}^{2}\right)^{-1}(\alpha, \phi) N_{l}^{2}(\alpha, \phi) P_{2}\right\|_{\infty}\right\} e .
\end{aligned}
$$


which implies

$$
\begin{aligned}
& \left\|P_{1}^{-1}\left(H_{\mathrm{GUSAOR}}\right)_{k} P_{1}\right\|_{\infty} \\
& \leq \max _{1 \leq l \leq s}\left\{\left\|P_{1}^{-1}\left(M_{l}^{1}\right)^{-1}(\beta, \psi) N_{l}^{1}(\beta, \psi) P_{1}\right\|_{\infty}\right. \\
& \left.\quad \times\left\|P_{2}^{-1}\left(M_{l}^{2}\right)^{-1}(\alpha, \phi) N_{l}^{2}(\alpha, \phi) P_{2}\right\|_{\infty}\right\} \\
& <1, \\
& \left|\left(H_{\mathrm{GUSAOR}}\right)_{k}\right| P_{1} e \\
& =P_{1}\left(P_{1}^{-1}\left|H_{\mathrm{GUSAOR}}(\alpha, \beta, \phi, \psi)_{k}\right| P_{1}\right) e \\
& \leq P_{1}\left\|P_{1}^{-1} H_{\mathrm{GUSAOR}}(\alpha, \beta, \phi, \psi)_{k} P_{1}\right\|_{\infty} e \\
& \leq \max _{1 \leq l \leq s}\left\{\left\|P_{1}^{-1}\left(M_{l}^{1}\right)^{-1}(\beta, \psi) N_{l}^{1}(\beta, \psi) P_{1}\right\|_{\infty}\right. \\
& \left.\quad \times\left\|P_{2}^{-1}\left(M_{l}^{2}\right)^{-1}(\alpha, \phi) N_{l}^{2}(\alpha, \phi) P_{2}\right\|_{\infty}\right\} P_{1} e .
\end{aligned}
$$

If we define

$$
\begin{aligned}
\sigma=\max _{1 \leq l \leq s}\left\{\left\|P_{1}^{-1}\left(M_{l}^{1}\right)^{-1}(\beta, \psi) N_{l}^{1}(\beta, \psi) P_{1}\right\|_{\infty}\right. \\
\left.\times\left\|P_{2}^{-1}\left(M_{l}^{2}\right)^{-1}(\alpha, \phi) N_{l}^{2}(\alpha, \phi) P_{2}\right\|_{\infty}\right\} .
\end{aligned}
$$

Then we have

$$
\left\|\left(H_{\mathrm{GUSAOR}}\right)_{k}\right\| \leq \sigma<1 .
$$

According to Algorithm 7, we can also get the following result.

Theorem 12. Let $A \in R^{n \times n}$ be an $H$-matrix and $\left(I-L_{l}, U_{l}, E_{l}\right)$, $l=1,2, \ldots, s$, a multisplitting of A. Assume that for $l=$ $1,2, \ldots, s$, we have the following.

(1) $L_{l}$ are the strictly lower triangular matrices and $U_{l}$ are the matrices such that the equalities $A=I-L_{l}-U_{l}$ hold.

(2) $\langle A\rangle=|I|-\left|L_{l}\right|-\left|U_{l}\right|=|I|-|B|$, where $|B|=\left|L_{l}\right|+\left|U_{l}\right|$.

(3) $P$ is diagonal matrix defined in Lemma 3 and $M_{l}^{1}(\beta, \psi), \quad N_{l}^{1}(\beta, \psi), \quad M_{l}^{2}(\alpha, \phi)$, and $N_{l}^{2}(\alpha, \phi)$ in Theorem 11 .

Then the sequence $\left\{x^{(k)}\right\}$ generated by Algorithm 7 converges for any initial $x^{(0)}$ if and only if $\left(\alpha, \beta, \beta_{i}, \gamma_{i}, \omega\right)$, $i=1,2, \ldots, n \in W_{2}$, with $\rho=(|B|)$ and $\theta=$ $\max _{1 \leq l \leq s}\left\{\left\|P_{1}^{-1}\left(M_{l}^{1}\right)^{-1}(\beta, \psi) N_{l}^{1}(\beta, \psi) P_{1}\right\|_{\infty} \| P_{2}^{-1}\left(M_{l}^{2}\right)^{-1}\right.$ $\left.(\alpha, \phi) N_{l}^{2}(\alpha, \phi) P_{2} \|_{\infty}\right\}$, where

$$
\begin{gathered}
W_{2}=\left\{\left(\alpha, \beta, \beta_{i}, \gamma_{i}, \omega\right) \in R^{5}: 0 \leq \alpha, \beta \leq 1,0<\beta_{i},\right. \\
\left.\gamma_{i}<\frac{2}{1+\rho}, 0<\omega<\frac{2}{1+\theta}, i=1,2, \ldots, n .\right\} .
\end{gathered}
$$

Proof. Define the iteration matrix in Algorithm 7

$$
\left(H_{\mathrm{GUSAOR}}\right)_{k}=\omega\left(H_{\mathrm{GUSAOR}}\right)_{k}+(1-\omega) I,
$$

where

$$
\begin{aligned}
\left(H_{\mathrm{GUSAOR}}\right)_{k} & =\sum_{l=1}^{s} E_{l}\left\{H(\beta, \psi)_{l} H(\alpha, \phi)_{l}\right\}^{\mu_{l, k}} . \\
H(\alpha, \phi)_{l} & =\left(I-\alpha \phi L_{l}\right)^{-1}\left[I-\phi+(1-\alpha) \phi L_{l}+\phi U_{l}\right], \\
H(\beta, \psi)_{l} & =\left(I-\beta \psi U_{l}\right)^{-1}\left[I-\psi+(1-\beta) \psi U_{l}+\psi L_{l}\right] .
\end{aligned}
$$

Obviously, similar to Theorem 11 we only need to find a constant $\sigma$ with $0 \leq \sigma<1$, which is independent of $k$, such that $K=\left\|P_{1}^{-1} H_{\text {GUSAOR }}(\alpha, \beta, \phi, \psi, \omega)_{k} P_{1}\right\|_{\infty} \leq \sigma$. From the proof of Theorem 11, we can get

$$
\begin{aligned}
K \leq & \omega\left\|P_{1}^{-1}\left(H_{\text {GUSAOR }}\right)_{k} P_{1}\right\|_{\infty}+|1-\omega| \\
\leq \omega \max _{1 \leq l \leq s} & \left\{\left\|P_{1}^{-1}\left(M_{l}^{1}\right)^{-1}(\beta, \psi) N_{l}^{1}(\beta, \psi) P_{1}\right\|_{\infty}\right. \\
& \left.\quad \times\left\|P_{2}^{-1}\left(M_{l}^{2}\right)^{-1}(\alpha, \phi) N_{l}^{2}(\alpha, \phi) P_{2}\right\|_{\infty}\right\} \\
& +|1-\omega| \\
= & \omega \theta+|1-\omega| .
\end{aligned}
$$

Now let $\sigma$ denote the last item in above inequalities. Clearly, if $0<\beta_{i}<2 /(1+\rho), 0<\gamma_{i}<2 /(1+\rho), 0<\omega<2 /(1+\theta)$, then

$$
\left\|\left(H_{\mathrm{GUSAOR}}\right)_{k}\right\| \leq \sigma<1 .
$$

Using the proving process of Theorems 11 and 12 and [13, Theorem 2.8] we have the following convergence result about Algorithm 9.

Theorem 13. Let $A \in R^{n \times n}$ be an $H$-matrix and $\left(I-L_{l}, U_{l}, E_{l}\right)$, $l=1,2, \ldots, s$, a multisplitting of $A$. Assume that for $l=$ $1,2, \ldots, s$, we have the following.

(1) $L_{l}$ are the strictly lower triangular matrices and $U_{l}$ are the matrices such that the equalities $A=I-L_{l}-U_{l}$ hold.

(2) $\langle A\rangle=|I|-\left|L_{l}\right|-\left|U_{l}\right|=|I|-|B|$, where $|B|=\left|L_{l}\right|+\left|U_{l}\right|$.

(3) $P$ is diagonal matrix defined in Lemma 3 and $M_{l}^{1}(\beta, \psi), \quad N_{l}^{1}(\beta, \psi), \quad M_{l}^{2}(\alpha, \phi)$ and $N_{l}^{2}(\alpha, \phi) \quad$ in Theorem 11.

(4) The index sequence $\left\{P_{i}\right\}$ is admissible and regulated.

Then the sequence $\left\{x^{(k)}\right\}$ generated by Algorithm 9 converges for any initial $x^{(0)}$ if and only if $\left(\alpha, \beta, \beta_{i}, \gamma_{i}, \omega\right)$, $i=1,2, \ldots n \in W_{3}$, with $\rho=(|B|)$ and 
$\theta=\max _{1 \leq l \leq s}\left\{\left\|P_{1}^{-1}\left(M_{l}^{1}\right)^{-1}(\beta, \psi) N_{l}^{1}(\beta, \psi) P_{1}\right\|_{\infty} \| P_{2}^{-1}\left(M_{l}^{2}\right)^{-1}\right.$

$\left.(\alpha, \phi) N_{l}^{2}(\alpha, \phi) P_{2} \|_{\infty}\right\}$, where

$$
\begin{array}{r}
W_{3}=\left\{\left(\alpha, \beta, \beta_{i}, \gamma_{i}, \omega\right) \in R^{5}: 0 \leq \alpha, \beta \leq 1,0<\beta_{i},\right. \\
\left.\gamma_{i}<\frac{2}{1+\rho}, 0<\omega<\frac{2}{1+\theta}, i=1,2, \ldots, n .\right\} .
\end{array}
$$

Remark 14. It is known that an $M$-matrix or a symmetric positive definite $L$-matrix is also $H$-matrix. Therefore, the convergence results in Theorems 11, 12, and 13 are valid.

Remark 15. Since a strictly or an irreducible diagonally dominant matrix is also satisfying the condition of Theorems 11, 12 , and 13, our methods are also valid for these kinds of matrices. Furthermore, for strictly or irreducible diagonally dominant matrices, $\|B\|_{\infty}$ can take the place of $\rho$ in Theorems 11, 12 , and 13.

\section{Applied Convergence of Relaxed Chaotic Methods}

In Theorems 12 and 13 of this paper, we find that $\theta$ is difficult to compute when carrying out numerical experiments. So for irreducible diagonally dominant matrices, we also have the following applied results of convergence according to Algorithms 7 and 9, respectively.

Theorem 16. Let $A \in R^{n \times n}$ be an irreducible diagonally dominant matrix and $\left(I-L_{l}, U_{l}, E_{l}\right), l=1,2, \ldots, s, a$ multisplitting of $A$. Assume that for $l=1,2, \ldots, s$, we have the following.

(1) $L_{l}$ are the strictly lower triangular matrices and $U_{l}$ are the matrices such that the equalities $A=I-L_{l}-U_{l}$ hold.

(2) $\langle A\rangle=|I|-\left|L_{l}\right|-\left|U_{l}\right|=|I|-|B|$, where $|B|=\left|L_{l}\right|+\left|U_{l}\right|$.

(3) $P$ is diagonal matrix defined in Lemma 3 and $M_{l}^{1}(\beta, \psi), \quad N_{l}^{1}(\beta, \psi), \quad M_{l}^{2}(\alpha, \phi)$, and $N_{l}^{2}(\alpha, \phi)$ in Theorem 11 .

Then the sequence $\left\{x^{(k)}\right\}$ generated by Algorithm 7 converges for any initial $x^{(0)}$ if and only if $\left(\alpha, \beta, \beta_{i}, \gamma_{i}, \omega\right), i=1,2, \ldots, n \in$ $W_{4}$, with $\theta^{\prime}=\max _{1 \leq l \leq n}\left\{\left(\left|1-\gamma_{i}\right|+\gamma_{i}\|B\|_{\infty}\right)\right\} \max _{1 \leq l \leq n}\left\{\left(\left|1-\beta_{i}\right|+\right.\right.$ $\left.\left.\beta_{i}\|B\|_{\infty}\right)\right\}$, where

$$
\begin{gathered}
W_{4}=\left\{\left(\alpha, \beta, \beta_{i}, \gamma_{i}, \omega\right) \in R^{5}: 0 \leq \alpha, \beta \leq 1,0<\beta_{i},\right. \\
\left.\gamma_{i}<\frac{2}{1+\|B\|_{\infty}}, 0<\omega<\frac{2}{1+\theta^{\prime}}\right\} .
\end{gathered}
$$

Proof. We only prove $\left|\left(H_{\mathrm{GUSAOR}}\right)_{k}\right| x<\theta^{\prime \prime} x(k=1,2, \ldots, 0 \leq$ $\left.\theta^{\prime} \leq 1\right)$. Let us define the iteration matrix in Algorithm 7

$$
\left(H_{\mathrm{GUSAOR}}\right)_{k}=\omega\left(H_{\mathrm{GUSAOR}}\right)_{k}+(1-\omega) I .
$$

From the proving process of Theorem 11, we may define

$$
\begin{aligned}
& T_{1}=\left(I-\beta \psi\left|U_{l}\right|\right)^{-1}\left[|I-\psi|+(1-\beta) \psi\left|U_{l}\right|+\psi\left|L_{l}\right|\right], \\
& T_{2}=\left(I-\alpha \phi\left|L_{l}\right|\right)^{-1}\left[|I-\phi|+(1-\alpha) \phi\left|L_{l}\right|+\phi\left|U_{l}\right|\right] .
\end{aligned}
$$

At first, we need to prove $\rho\left(T_{1}\right)<1$. Similarly, we may also prove $\rho\left(T_{2}\right)<1$. Assume that

$$
\begin{gathered}
0 \leq \alpha, \quad \beta \leq 1, \\
0<\beta_{i}, \quad \gamma_{i}<\frac{2}{1+\rho} .
\end{gathered}
$$

With (36), we know that $T_{1}$ is nonnegative, so according to [24, Theorem 2.7], there exists an eigenvector $x \geq 0, x \neq 0$, such that $T_{1} x=\rho\left(T_{1}\right) x$ hold; that is,

$$
\left[|I-\psi|+(1-\beta) \psi\left|U_{l}\right|+\psi\left|L_{l}\right|\right] x=\rho\left(T_{1}\right)\left(I-\beta \psi\left|U_{l}\right|\right) x .
$$

Multiplying by $\psi^{-1}$, it holds that

$$
\left[\rho\left(T_{1}\right) \psi^{-1}-\left|I-\psi^{-1}\right|\right] x=\left\{\left[1-\beta+\beta \rho\left(T_{1}\right)\right]\left|U_{l}\right|+\left|L_{l}\right|\right\} x .
$$

As $\left[1-\beta+\beta \rho\left(T_{1}\right)\right]\left|U_{l}\right|+\left|L_{l}\right| \geq 0$, it follows by [5, Theorem 11] that

$$
\begin{aligned}
& \min _{1 \leq l \leq n}\left\{\gamma_{i}^{-1} \rho\left(T_{1}\right)-\left|1-\gamma_{i}^{-1}\right|\right\} \\
& \quad \leq \rho\left(\left[1-\beta+\beta \rho\left(T_{1}\right)\right]\left|U_{l}\right|+\left|L_{l}\right|\right) .
\end{aligned}
$$

From the proof of [3, Theorem 3.1], we have $\rho\left(T_{1}\right)<1$, $0 \leq 1-\beta+\beta \rho\left(T_{1}\right)<1$. Since the matrices $A, B$, and $\left[1-\beta+\beta \rho\left(T_{1}\right)\right]\left|U_{l}\right|+\left|L_{l}\right|$ are irreducible, by [24, Theorem 2.1], it follows that

$$
\begin{aligned}
\rho\left(\left[1-\beta+\beta \rho\left(T_{1}\right)\right]\left|U_{l}\right|+\left|L_{l}\right|\right) & <\rho\left(\left|L_{l}\right|+\left|U_{l}\right|\right) \\
& =\rho(|B|) \leq\|B\|_{\infty} .
\end{aligned}
$$

With (40) and (41) and $M$ being irreducible diagonally dominant by rows, we have

$$
\rho\left(T_{1}\right)<\max _{1 \leq l \leq n}\left\{\left|1-\gamma_{i}\right|+\gamma_{i}\|B\|_{\infty}\right\} .
$$

Notice that if $\gamma_{i} \leq 1$, we may obtain

$$
\left|1-\gamma_{i}\right|+\gamma_{i}\|B\|_{\infty}=1-\gamma_{i}+\gamma_{i}\|B\|_{\infty}<1 .
$$

While if $1<\gamma_{i}<2 /\left(1+\|B\|_{\infty}\right)$, we also have

$$
\begin{aligned}
\left|1-\gamma_{i}\right|+\gamma_{i}\|B\|_{\infty} & =-1+\gamma_{i}+\gamma_{i}\|B\|_{\infty} \\
& =\gamma_{i}\left(1+\|B\|_{\infty}\right)-1 \\
& <\frac{2}{1+\|B\|_{\infty}}\left(1+\|B\|_{\infty}\right)-1 \\
& =1,
\end{aligned}
$$


From (42), (43), and (44), we can get

$$
\begin{aligned}
& \rho\left(T_{1}\right)<\max _{1 \leq l \leq n}\left\{\left|1-\gamma_{i}\right|+\gamma_{i}\|B\|_{\infty}\right\}<1, \\
& \rho\left(T_{2}\right)<\max _{1 \leq l \leq n}\left\{\left|1-\beta_{i}\right|+\beta_{i}\|B\|_{\infty}\right\}<1 .
\end{aligned}
$$

From Theorem 11, we have

$$
\begin{aligned}
\left(H_{\mathrm{GUSAOR}}\right)_{k} & \leq\left|\left(H_{\mathrm{GUSAOR}}\right)_{k}\right| \\
& =\left|\sum_{l=1}^{s} H(\beta, \psi)_{l} H(\alpha, \phi)_{l}\right| \\
& \leq \sum_{l=1}^{s}\left|H(\beta, \psi)_{l}\right|\left|H(\alpha, \phi)_{l}\right| \\
& \leq \sum_{l=1}^{s} T_{1} T_{2} .
\end{aligned}
$$

From (35) and the above proof, we have

$$
\begin{aligned}
&\left(H_{\mathrm{GUSAOR}}\right)_{k} x \\
& \leq \omega\left|\left(H_{\mathrm{GUSAOR}}\right)_{k}\right| x+|1-\omega| x \\
& \leq \omega\left|\sum_{l=1}^{s} E_{l} H(\beta, \psi)_{l} H(\alpha, \phi)_{l}\right| x+|1-\omega| x \\
& \leq \omega \sum_{l=1}^{s} E_{l}\left|H(\beta, \psi)_{l}\right|\left|H(\alpha, \phi)_{l}\right| x+|1-\omega| x \\
& \leq \sum_{l=1}^{s} E_{l} T_{1} T_{2} x+|1-\omega| x \\
&=\rho\left(T_{2}\right) \omega \sum_{l=1}^{s} E_{l} T_{1} x+|1-\omega| x \\
&= \omega \rho\left(T_{1}\right) \rho\left(T_{2}\right) \sum_{l=1}^{s} E_{l} x+|1-\omega| x \\
&= \omega \rho\left(T_{1}\right) \rho\left(T_{2}\right) x+|1-\omega| x \\
&<\omega \max _{1 \leq l \leq n}\left\{\left|1-\gamma_{i}\right|+\gamma_{i}\|B\|_{\infty}\right\} \\
& \times \max _{1 \leq l \leq n}\left\{\left|1-\beta_{i}\right|+\beta_{i}\|B\|_{\infty}\right\} x+|1-\omega| x \\
&(\omega|1-\omega|) x \\
& \\
& \\
& \\
& \\
&
\end{aligned}
$$

where $\theta^{\prime}=\max _{1 \leq l \leq n}\left\{\left|1-\gamma_{i}\right|+\gamma_{i}\|B\|_{\infty}\right\} \max _{1 \leq l \leq n}\left\{\left|1-\beta_{i}\right|+\right.$ $\left.\beta_{i}\|B\|_{\infty}\right\}, 0<\omega<2 /\left(1+\theta^{\prime}\right), \theta^{\prime \prime}=\omega \theta^{\prime}+|1-\omega|<1$.

Remark 17. Obviously, convergence results of Theorem 16 are convenient for carrying out numerical experiments. Using the proving process of Theorems 11 and 16 and [13, Theorem 2.8], we can get the following result about Algorithm 9 .
Theorem 18. Let $A \in R^{n \times n}$ be an irreducible diagonally dominant matrix and $\left(I-L_{l}, U_{l}, E_{l}\right), l=1,2, \ldots, s, a$ multisplitting of $A$. Assume that for $l=1,2, \ldots, s$, we have the following.

(1) $L_{l}$ are the strictly lower triangular matrices and $U_{l}$ are the matrices such that the equalities $A=I-L_{l}-U_{l}$ hold.

(2) $\langle A\rangle=|I|-\left|L_{l}\right|-\left|U_{l}\right|=|I|-|B|$, where $|B|=\left|L_{l}\right|+\left|U_{l}\right|$.

(3) $P$ is diagonal matrix defined in Lemma 3 and $M_{l}^{1}(\beta, \psi), \quad N_{l}^{1}(\beta, \psi), \quad M_{l}^{2}(\alpha, \phi)$, and $N_{l}^{2}(\alpha, \phi)$ in Theorem 11 .

(4) The index sequence $\left\{P_{i}\right\}$ is admissible and regulated.

Then the sequence $\left\{x^{(k)}\right\}$ generated by Algorithm 9 converges for any initial $x^{(0)}$ if and only if $\left(\alpha, \beta, \beta_{i}, \gamma_{i}, \omega\right), i=1,2, \ldots, n \in$ $W_{5}$, with $\theta^{\prime}=\max _{1 \leq l \leq n}\left\{\left(\left|1-\gamma_{i}\right|+\gamma_{i}\|B\|_{\infty}\right)\right\} \max _{1 \leq l \leq n}\left\{\left(\left|1-\beta_{i}\right|+\right.\right.$ $\left.\left.\beta_{i}\|B\|_{\infty}\right)\right\}$, where

$$
\begin{gathered}
W_{5}=\left\{\left(\alpha, \beta, \beta_{i}, \gamma_{i}, \omega\right) \in R^{5}: 0 \leq \alpha, \beta \leq 1,0<\beta_{i},\right. \\
\left.\gamma_{i}<\frac{2}{1+\|B\|_{\infty}}, 0<\omega<\frac{2}{1+\theta^{\prime}}\right\} .
\end{gathered}
$$

Remark 19. As a special case, for the relaxed matrix multisplitting chaotic GSSOR-style methods, we have the corresponding convergence results, where $\alpha=1, \beta=1, \phi=$ $\operatorname{diag}\left(\beta_{1}, \beta_{2}, \ldots, \beta_{n}\right), \psi=\operatorname{diag}\left(\gamma_{1}, \gamma_{2}, \ldots, \gamma_{n}\right)$ with $\beta_{i} \geq 0$, $\gamma_{i} \geq 0(i=1,2, \ldots, n)$ and $\omega$ real parameters.

\section{Numerical Examples}

Example 1. By using difference discretization of partial differential equation, we can obtain the corresponding coefficient matrix of the linear system $(n=6)$, which is as follows:

$$
A=\left[\begin{array}{cccccc}
1 & \frac{-1}{4} & 0 & 0 & 0 & 0 \\
1 & 1 & \frac{3}{4} & 0 & 0 & 0 \\
0 & \frac{1}{3} & 1 & \frac{-1}{3} & 0 & 0 \\
0 & 0 & \frac{3}{4} & 1 & 1 & 0 \\
0 & 0 & 0 & \frac{1}{4} & 1 & \frac{-1}{4} \\
0 & 0 & 0 & 0 & 1 & 1
\end{array}\right], \quad b=\left[\begin{array}{c}
\frac{3}{4} \\
\frac{11}{4} \\
3 \\
\frac{11}{4} \\
1 \\
2
\end{array}\right] .
$$

Now, we will apply the results of Theorem 16 according to Algorithm 7. From Algorithm 7, we can get the iterative matrix

$$
H=\omega \sum_{l=1}^{s} E_{l}\left[H(\beta, \psi)_{l} H(\alpha, \phi)_{l}\right]+(1-\omega) \text {. }
$$

Here, we assume that $s=3, \mu_{l, k} \equiv 1$. By direct calculations with Matlab 7.1, we have

$$
\|B\|_{\infty}=1.75, \frac{2}{1+\|B\|_{\infty}}=0.7273 .
$$


TABLE 1: Convergence results of Theorems 16 and 18.

\begin{tabular}{lcccc}
\hline$\left(\alpha, \beta, \beta_{i}, \gamma_{i}, \omega\right)$ & \multicolumn{2}{c}{$\frac{2}{1+\theta^{\prime}}$} & $\rho(H)$ \\
\cline { 2 - 3 } & $1+\|B\|_{\infty}$ & & 1.3793 & 0.5711 \\
$(0.2,0.8,0.6,0.5,1.1)$ & 0.7273 & & 1.3115 & 0.6133 \\
$(0.3,0.8,0.7,0.5,1.1)$ & 0.7273 & & 1.3793 & 0.8365 \\
$(0.4,0.7,0.6,0.5,0.8)$ & 0.7273 & & 1.3793 & 0.69393 \\
$(0.8,0.2,0.3,0.7,1.1)$ & 0.7273 & 1.3115 & 0.6053 \\
$(0.9,0.1,0.25,0.65,1.1)$ & 0.7273 & 1.3445 & 0.7154 \\
\hline
\end{tabular}

In Table 1, we show that convenience results of Section 4 are convenience for carrying out numerical experiments, where $\rho(H)$ denote the spectral radius of iterative matrix $H$ :

$$
\begin{gathered}
E_{1}=\operatorname{diag}(1,1,0,0,0,0), \quad E_{2}=\operatorname{diag}(0,0,1,1,0,0), \\
E_{3}=\operatorname{diag}(0,0,0,0,1,1) \\
L_{1}=\left[\begin{array}{cccccc}
0 & 0 & 0 & 0 & 0 & 0 \\
\frac{1}{2} & 0 & 0 & 0 & 0 & 0 \\
0 & \frac{1}{3} & 0 & 0 & 0 & 0 \\
0 & 0 & \frac{3}{20} & 0 & 0 & 0 \\
0 & 0 & 0 & \frac{1}{4} & 0 & 0 \\
0 & 0 & 0 & 0 & -0.4 & 0
\end{array}\right], \\
U_{l}=I-L_{l}-\frac{A}{2}, \frac{1}{2}= \\
L_{2}=\left[\begin{array}{ccccccc}
0 & 0 & 0 & 0 & 0 & 0 \\
0.3 & 0 & 0 & 0 & 0 & 0 \\
0 & 0.5 & 0 & 0 & 0 & 0 \\
0 & 0 & -0.3 & 0 & 0 & 0 \\
0 & 0 & 0 & 0 & 0 & 0 \\
0 & \frac{1}{6} & 0 & 0 & 0 & 0 \\
0 & 0 & \frac{7}{20} & 0 & 0 & 0 \\
0 & 0 & 0 & \frac{1}{8} & 0 & 0 \\
0 & 0 & 0 & 0 & 0.2 & 0
\end{array}\right],
\end{gathered}
$$

Remark 20. Obviously, $\theta^{\prime}$ of Theorems 16 and 18 is applied and easily calculated when carrying out numerical experiments.
Example 2. Consider a matrix $A \in R^{n \times n}$ of the form

$$
\begin{aligned}
A & =\left[\begin{array}{cccccc}
1 & 0 & 0 & \cdots & 0 & -v \\
-v & 1 & 0 & \cdots & 0 & 0 \\
0 & -v & 1 & \cdots & 0 & 0 \\
\vdots & \vdots & \ddots & \vdots & \vdots & \vdots \\
0 & 0 & 0 & \cdots & 1 & 0 \\
0 & 0 & 0 & \cdots & -v & 1
\end{array}\right] \\
= & {\left[\begin{array}{cccccc}
1 & 0 & 0 & \cdots & 0 & 0 \\
0 & 1 & 0 & \cdots & 0 & 0 \\
0 & 0 & 1 & \cdots & 0 & 0 \\
\vdots & \vdots & \ddots & \vdots & \vdots & \vdots \\
0 & 0 & 0 & \cdots & 1 & 0 \\
0 & 0 & 0 & \cdots & 0 & 1
\end{array}\right] } \\
& -\left[\begin{array}{cccccc}
0 & 0 & 0 & \cdots & 0 & \nu \\
\nu & 0 & 0 & \cdots & 0 & 0 \\
0 & \nu & 0 & \cdots & 0 & 0 \\
\vdots & \vdots & \ddots & \vdots & \vdots & \vdots \\
0 & 0 & 0 & \cdots & 0 & 0 \\
0 & 0 & 0 & \cdots & v & 0
\end{array}\right]
\end{aligned}
$$

where $\rho\left(|D|^{-1}|B|\right)=\nu, v$ is a real number, satisfying $|\nu|<1$.

In Theorem 11, let $0 \leq \alpha, \beta \leq 1,0<\beta_{i}, \gamma_{i}<2 /(1+\nu), i=$ $1,2, \ldots, n$; then Algorithm 5 converges for any initial vector $x^{(0)}$. In Theorems 12 and 13, let $0 \leq \alpha, \beta \leq 1,0<\beta_{i}, \gamma_{i}<$ $2 /(1+\nu), 0<\omega<2 /(1+\theta), i=1,2, \ldots, n$; then Algorithms 7 and 9 converge for any initial vector $x^{(0)}$. In Theorems 16 and 18 , let $0 \leq \alpha, \beta \leq 1,0<\beta_{i}, \gamma_{i}<2 /(1+\nu), 0<\omega<$ $2 /\left(1+\theta^{\prime}\right), i=1,2, \ldots, n$; then Algorithms 7 and 9 converge for any initial vector $x^{(0)}$, where $\theta^{\prime}=\max _{1 \leq l \leq n}\left\{\left(\left|1-\gamma_{i}\right|+\right.\right.$ $\left.\left.\nu \gamma_{i}\right)\right\} \max _{1 \leq l \leq n}\left\{\left(\left|1-\beta_{i}\right|+\nu \beta_{i}\right)\right\}$.

If we choose $\nu=1 / 5$, in Theorem 11 , let $0 \leq \alpha, \beta \leq 1$, $0<\beta_{i}, \gamma_{i}<5 / 3, i=1,2, \ldots, n$; then Algorithm 5 converges for any initial vector $x^{(0)}$. In Theorems 12 and 13, let $0 \leq \alpha$, $\beta \leq 1,0<\beta_{i}, \gamma_{i}<5 / 3,0<\omega<2 /(1+\theta), i=1,2, \ldots, n$; then Algorithms 7 and 9 converge for any initial vector $x^{(0)}$. In Theorems 16 and 18, let $0 \leq \alpha, \beta \leq 1,0<\beta_{i}, \gamma_{i}<5 / 3$, $0<\omega<2 /\left(1+\theta^{\prime}\right), i=1,2, \ldots, n$; then Algorithms 7 and 9 converge for any initial vector $x^{(0)}$, where $\theta^{\prime}=\max _{1 \leq l \leq n}\{(\mid 1-$ $\left.\left.\gamma_{i} \mid+(1 / 5) \gamma_{i}\right)\right\} \max _{1 \leq l \leq n}\left\{\left(\left|1-\beta_{i}\right|+(1 / 5) \beta_{i}\right)\right\}$.

\section{Conclusions}

In this paper, we consider relaxed matrix parallel multisplitting chaotic GUSAOR-style methods for solving linear systems of algebraic equations $A x=b$, in which the coefficient $A$ is an $H$-matrix or an irreducible diagonally dominant matrices, and analyze the convergence of our methods, which use more relaxed factors and are the generalization of $[11,13,14]$. The parameters can be adjusted suitably so that the convergence property of method can be substantially improved. Furthermore, we further study some applied convergence results of methods to be convenient for carrying out numerical experiments. Finally, we give some 
applied examples, which show that our convergence results are applied and easily calculated when carrying out numerical experiments.

Particularly, one may discuss how to choose the set of relaxed parameters in order to really accelerate the convergence of the considered method. Furthermore, The optimal choice of this set of relaxed parameters is valuably studied.

\section{Conflict of Interests}

The authors declare that there is no conflict of interests regarding the publication of this paper.

\section{Acknowledgments}

This research of this author is supported by NSFC Tianyuan Mathematics Youth Fund (11226337), NS-FC (61203179, 61202098, 61170309, 91130024, 61033009, 61272544, and 11171039), Aeronautical Science Foundation of China (2013ZD55006), Project of Youth Backbone Teachers of Colleges and Universities in Henan Province (2013GGJS142), ZZIA Innovation team fund (2014TD02), Major project of development foundation of science and technology of CAEP (2012A0202008), Basic and Advanced Technological Research Project of Henan Province (132300410373, 142300410333), and Natural Science Foundation of Henan Province (14B110023).

\section{References}

[1] D. P. O'Leary and R. E. White, "Multisplittings of matrices and parallel solution of linear systems," SIAM Journal on Algebraic and Discrete Methods, vol. 6, no. 4, pp. 630-640, 1985.

[2] A. Frommer and G. Mayer, "Convergence of relaxed parallel multisplitting methods," Linear Algebra and Its Applications, vol. 119, pp. 141-152, 1989.

[3] J. Mas, V. Migallón, J. Penadés, and D. B. Szyld, "Nonstationary parallel relaxed multisplitting methods," Linear Algebra and Its Applications, vol. 241-243, pp. 733-747, 1992.

[4] T. X. Gu, X. P. Liu, and L. J. Shen, "Relaxed parallel two-stage multisplitting methods," International Journal of Computer Mathematics, vol. 75, no. 3, pp. 351-367, 2000.

[5] T.-X. Gu and X.-P. Liu, "Parallel two-stage multisplitting iterative methods," International Journal of Computer Mathematics, vol. 20, no. 2, pp. 153-166, 1998.

[6] D. Chazan and W. Miranker, "Chaotic relaxation," Linear Algebra and its Applications, vol. 2, pp. 199-222, 1969.

[7] R. Bru, L. Elsner, and M. Neumann, "Models of parallel chaotic iteration methods," Linear Algebra and its Applications, vol. 103, pp. 175-192, 1988.

[8] L. Elsner, M. Neumann, and B. Vemmer, "The effect of the number of processors on the convergence of the parallel block Jacobi method," Linear Algebra and Its Applications, vol. 154156, pp. 311-330, 1991.

[9] D. Jiang, Z. Xu, Z. Chen, Y. Han, and H. Xu, "Joint timefrequency sparse estimation of large-scale network traffic," Computer Networks, vol. 55, no. 15, pp. 3533-3547, 2011.

[10] D.-D. Jiang, Z.-Z. Xu, H.-W. Xu, Y. Han, Z.-H. Chen, and Z. Yuan, "An approximation method of origin-destination flow traffic from link load counts," Computers \& Electrical Engineering, vol. 37, no. 6, pp. 1106-1121, 2011.
[11] P. E. Kloeden and D. J. Yuan, "Convergence of relaxed chaotic parallel iterative methods," Bulletin of the Australian Mathematical Society, vol. 50, no. 1, pp. 167-176, 1994.

[12] S.-Q. Shen and T.-Z. Huang, "New comparison results for parallel multisplitting iterative methods," Applied Mathematics and Computation, vol. 206, no. 2, pp. 738-747, 2008.

[13] Y. Song and D. Yuan, "On the convergence of relaxed parallel chaotic iterations for $H$-matrix," International Journal of Computer Mathematics, vol. 52, no. 3-4, pp. 195-209, 1994.

[14] D. Yuan, "On the convergence of parallel multisplitting asynchronous GAOR method for $H$-matrix," Applied Mathematics and Computation, vol. 160, no. 2, pp. 477-485, 2005.

[15] L. T. Zhang, T. Z. Huang, T. X. Gu, and X. L. Guo, "Convergence of relaxed multisplitting USAOR methods for $H$-matrices linear systems," Applied Mathematics and Computation, vol. 202, no. 1, pp. 121-132, 2008.

[16] L. Zhang, T. Huang, T. Gu, X. Guo, and J. Yue, "Convergent improvement of SSOR multisplitting method for an $H$-matrix," Journal of Computational and Applied Mathematics, vol. 225, no. 2, pp. 393-397, 2009.

[17] L.-T. Zhang, T.-Z. Huang, S.-H. Cheng, T.-X. Gu, and Y.-P. Wang, "A note on parallel multisplitting TOR method for $H$ matrices," International Journal of Computer Mathematics, vol. 88, no. 3, pp. 501-507, 2011.

[18] L. T. Zhang, T. Z. Huang, S. H. Cheng, and T. X. Gu, "The weaker convergence of non-stationary matrix multisplitting methods for almost linear systems," Taiwanese Journal of Mathematics, vol. 15, no. 4, pp. 1423-1436, 2011.

[19] L.-T. Zhang and J.-L. Li, "The weaker convergence of modulusbased synchronous multisplitting multi-parameters methods for linear complementarity problems," Computers \& Mathematics with Applications, vol. 67, no. 10, pp. 1954-1959, 2014.

[20] L.-T. Zhang and X.-Y. Zuo, "Improved convergence theorems of multisplitting methods for the linear complementarity problem," Applied Mathematics and Computation. In press.

[21] L.-T. Zhang, "A new preconditioner for generalized saddle matrices with highly singular(1,1) blocks," International Journal of Computer Mathematics, 2013.

[22] Y. Zhang, "The USAOR iterative method for linear systems," Numerical Mathematics, vol. 9, no. 4, pp. 354-365, 1987 (Chinese).

[23] Y. Song, "On the convergence of the generalized AOR method," Linear Algebra and Its Applications, vol. 256, pp. 199-218, 1997.

[24] R. S. Varga, Matrix Iterative Analysis, Prentice Hall, Englewood Cliffs, NJ, USA, 1962. 


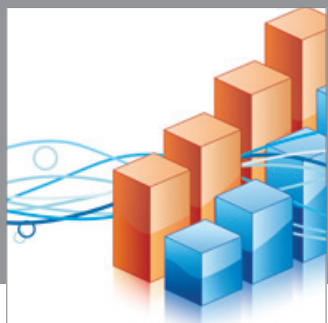

Advances in

Operations Research

mansans

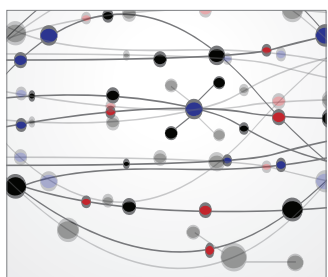

The Scientific World Journal
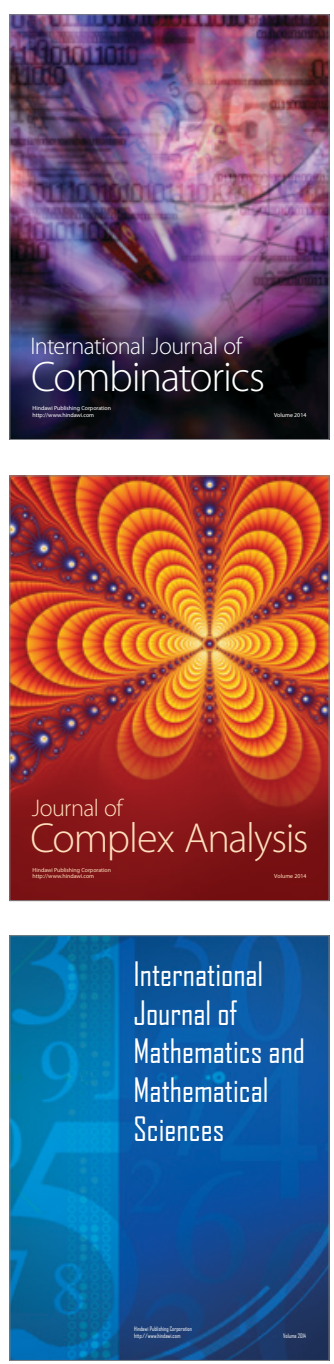
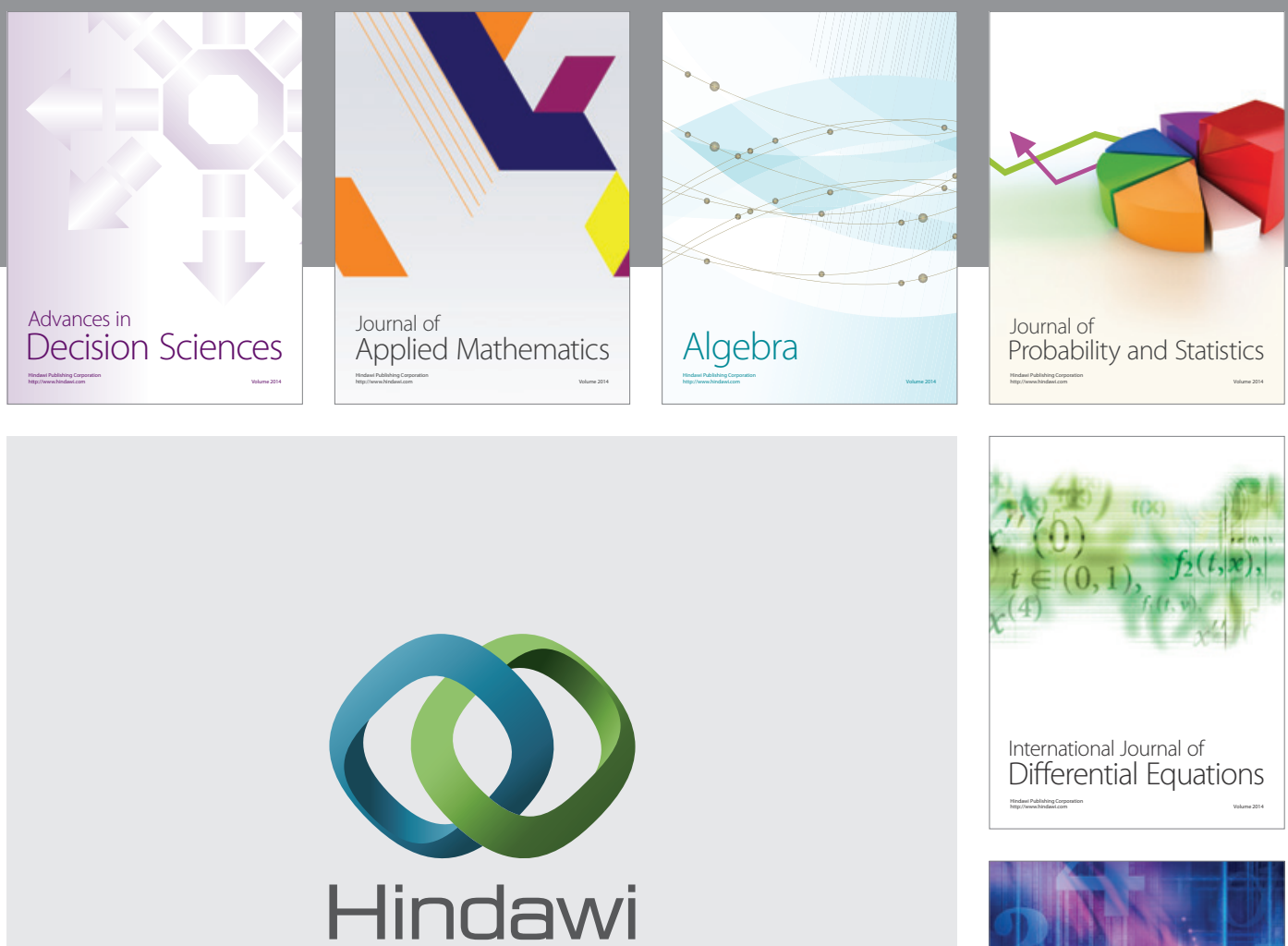

Submit your manuscripts at http://www.hindawi.com
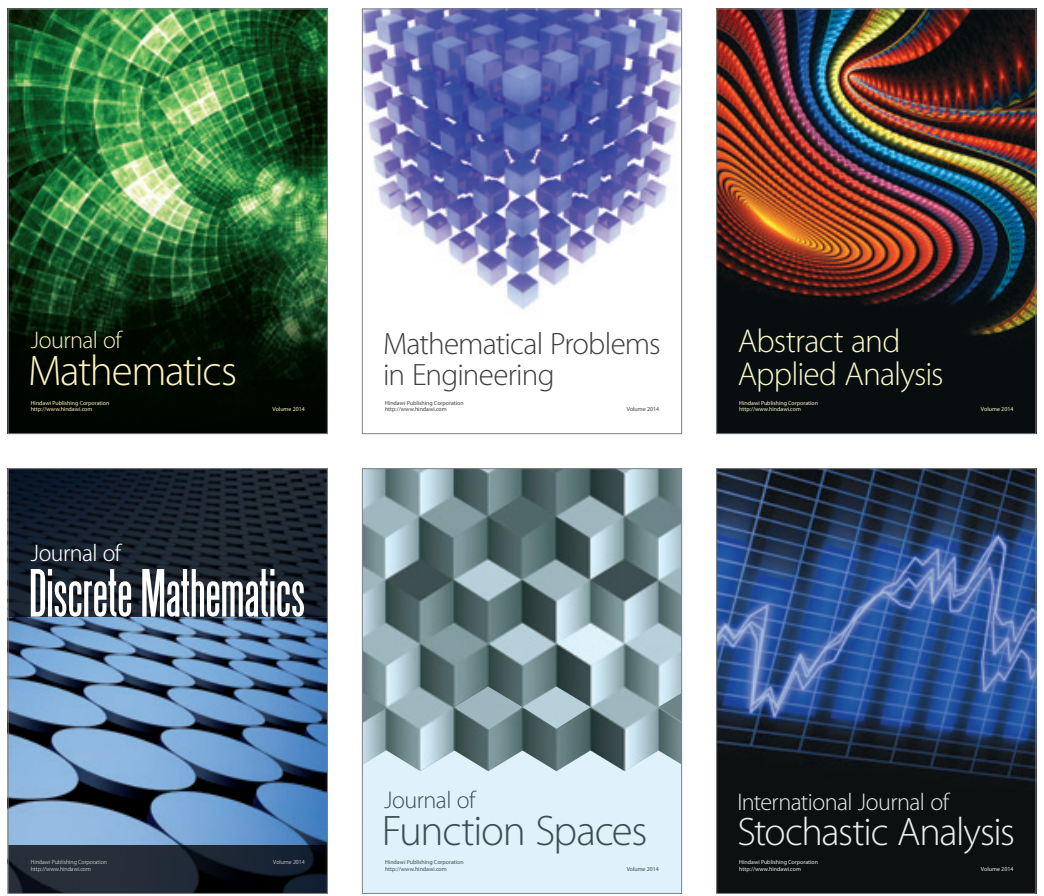

Journal of

Function Spaces

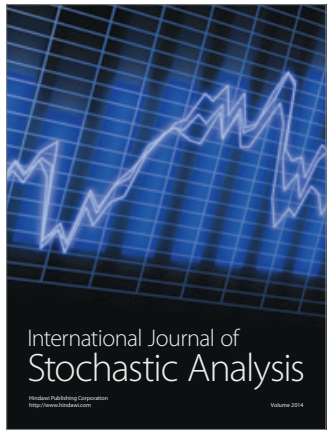

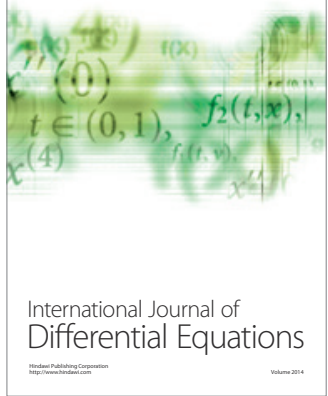
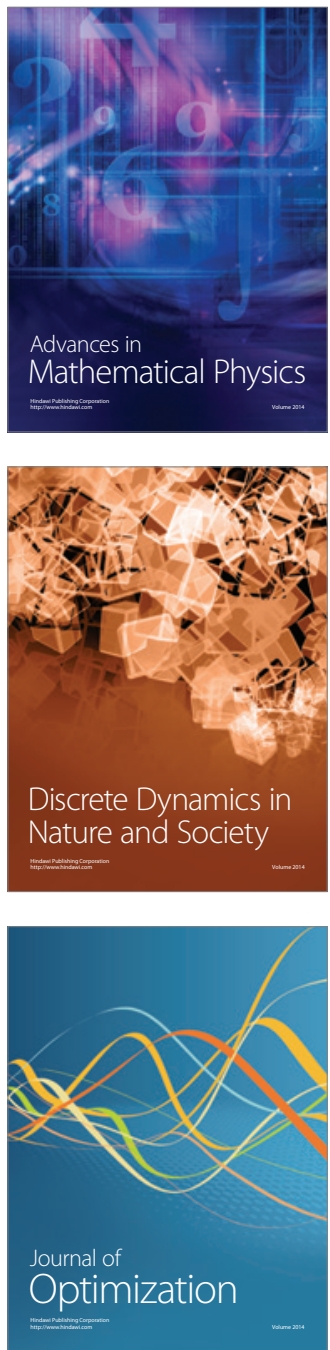